\title{
Category of Metabolic-Replication Systems in Biology and Medicine
}

$03 / 31 / 2012$

\author{
I.C. Baianu \\ AFC-NMR \& NIR Microspectroscopy Facility, \\ College of ACES, FSHN \& NPRE Departments, \\ University of Illinois at Urbana, \\ Urbana, IL. 61801, USA \\ Email address: ibaianu @illinois.edu
}

\section{Introduction to Abstract Metabolic-Replication Systems}

Robert Rosen introduced metabolic-repair models, or (M,R)-systems in mathematical biology (abstract relational biology) in 1957 ([4,$\underline{5}])$; such systems will be here abbreviated as $M R$-systems, (or simply $M R$ 's). Rosen, then represented the $M R$ 's in terms of categories of sets, deliberately selected without any structure other than the discrete topology of sets.

Theoreticians of life's origins postulate that Life on Earth has begun with the simplest possible organism, called the primordial. Mathematicians interested in biology and this important question of the minimal living organism have attempted to define the functional relations that would have made life possible in such a minimal system- a grandad and granma of all living organisms on Earth.

Definition 1.1 The simplest $M R$-system is a relational model of the primordial organism which is defined by the following categorical sequence (or diagram) of sets and set-theoretical

mappings:

$$
f: A \rightarrow B, \phi: B \rightarrow \operatorname{Hom}_{M R}(A, B)
$$

, where $A$ is the set of inputs 
to the $M R$-system, $B$ is the set of its outputs, and is the 'repair map', or $R$-component, of the $M R$-system which associates to a certain product, or output $b$, the 'metabolic' component (such as an enzyme, E, for example)

represented by the set-theoretical mapping ${ }^{f}$. Then, $\operatorname{Hom}_{M R}(A, B)$ is defined as the set of all such metabolic (set-theoretical) mappings

(occasionally written incorrectly by some authors as

Definition 1.2 A general -system was defined by Rosen (1958a,b) as the network or graph of the metabolic and repair components that were specified above inDefinition 0.1 ; such components are networked in a complex, abstract 'organism' defined by all the abstract relations and connecting maps between the sets specifying all the metabolic and repair components of such a general, abstract model of the biological organism. The $(M, R)$

mappings bettwen -systems are defined as the the metabolic and repair set-theoretical mappings, such as ${ }^{f}$ and ${ }^{\phi}$ (specified inDefinition 0.1); moreover, there is also a finite number of sets (just like those that are defined as in Definition 0.1): ${ }^{A_{i}, B_{i}}$, whereas $f \in \operatorname{Hom}_{M R_{i}}\left(A_{i}, B_{i}\right)$ and $\phi \in \operatorname{Hom}_{M R_{i}}\left[B, \operatorname{Hom}_{M R_{i}}\left(A_{i}, B_{i}\right)\right]$, with ${ }^{i \in I}$, and $I$ being a finite index set, or directed set, with being a finite number of distinct metabolic and repair components pairs. Alternatively, one may think of a a general $M R$ system as being 'made of' a finite number $N$ of interconnected $M R_{i}$ metabolic-repair modules with input sets ${ }^{A_{i}}$ and output sets ${ }^{B_{i}}$. To sum up: a general MR-system can be defined as a family of interconnected quartets: $\left\{\left(A_{i}, B_{i}, f_{i}, \phi_{i}\right)\right\}_{i \in I}$, where $I$ is an index set of

\section{Category of $(M, R)$-systems}

\section{Definition 1.3}



A category of
$(M, R)$
-system quartet modules,
$\left\{\left(A_{i}, B_{i}, f_{i}, \phi_{i}\right)\right\}_{i \in I}$, with I
being an index set of integers $i=1,2, \ldots, n$
set-theoretical mappings defined by the MR-morphisms between the quarted modules $\left\{\left(A_{i}, B_{i}, f_{i}, \phi_{i}\right)\right\}_{i \in I}$
defined as $\phi_{i} \in \operatorname{Hom}_{M R_{i}}\left[B, \operatorname{Hom}_{M R_{i}}\left(A_{i}, B_{i}\right)\right]$
composition defined by the usual composition of functions between sets.
With a few, additional notational changes it can be shown that the category $(M, R)$
of -systems is a subcategory of the category of automata (or sequential machines), ${ }^{\mathcal{S}_{[M, A]}}([\underline{7}, \underline{8}])$.

Remark 1.1 For over two decades, Robert Rosen developed with several coworkers the MR-systems theory and its applications to life sciences, medicine and general systems theory. He also considered biocomplexity to be an 'emergent', defining feature of organisms which is not reducible in terms of the molecular structures (or molecular components) of the organism and their physicochemical interactions. However, in his last written book in 1997 on "Essays on Life Itself", published posthumously in 2000, Robert Rosen finally accepted the need for representing organisms in terms of categories with structure that entail biological functions, both metabolic and repair ones. Note also that, unlike Rashevsky in his theory of organismic sets, Rosen did not attempt to extend the $M R$ s to modeling societies, even though with

appropriate modifications of generalized $(M, R)$ structure $([\underline{7}, \underline{8}, 13])$, this is feasible and yields meaningful mathematical and sociological results. Thus, subsequent publications have generalized MRsystem (GMRs) and have studied the fundamental, mathematical properties of algebraic categories of GMRs that were constructed functorially based on the Yoneda-Grothendieck Lemma and construction. Then it was shown that such algebraic categories of GMRs are Cartesian closed [7]. Several molecular biology realizations of GMRs in terms of DNA, RNAs, enzymes, $R N A \rightarrow D N A$-reverse trancriptases, and other biomolecular components were subsequently introduced and discussed in ref. $[\underline{21}, \underline{13}, 14]$ in 
terms of non-linear genetic network models in many-valued, ${ }^{L M_{n}}$ logic algebras (or algebraic category $\mathcal{L} \mathcal{M}$ of ${ }^{L} \quad$ logic algebras).
If simple
$(M, R)$
automata the category of
-systems are considered as sequential machines or
homomorphisms is a subcategory of the automata category. However,
$(M, R)$
-systems and
$(M, R)$
-system
when $\quad$-systems are considered together with their dynamic
representations the category of dynamic $(M, R)$ subcategory of the category of automata.
-systems is no longer a

\section{Bibliography}

Rashevsky, N.: 1965, The Representation of Organisms in Terms of Predicates, Bulletin of Mathematical Biophysics 27: 477-491.

Rashevsky, N.: 1969, Outline of a Unified Approach to Physics, Biology and Sociology., Bulletin of Mathematical Biophysics 31: 159-198.

Rosen, R.: 1985, Anticipatory Systems, Pergamon Press: New York.

Rosen, R.: 1958a, A Relational Theory of Biological Systems Bulletin of Mathematical Biophysics 20: 245-260.

Rosen, R.: 1958b, The Representation of Biological Systems from the Standpoint of the Theory of Categories., Bulletin of Mathematical Biophysics 20: 317-341.

6

Rosen, R.: 1987, On Complex Systems, European Journal of Operational Research 30:129-134.

Baianu, I.C.: 1973, Some Algebraic Properties of $(M, R)$ -

Systems. Bulletin of Mathematical Biophysics 35, 213-217. 
Baianu, I.C. and M. Marinescu: 1974, On A Functorial Construction of $(M, R)$ - Systems. Revue Roumaine de Mathematiques Pures et Appliquées 19: 388-391.

9 10

Baianu, I.C.: 2004a. Łukasiewicz-Topos Models of Neural Networks,

Baianu, I.C.: 2004a. Łukasiewicz-Topos Models of Neural Network Eprint. Cogprints-Sussex Univ.

Baianu, I.C.: 1980, Natural Transformations of Organismic Structures., Bulletin of Mathematical Biology,42: 431-446.

I.C. Baianu: 1977, A Logical Model of Genetic Activities in Łukasiewicz Algebras: The Non-linear Theory. Bulletin of Mathematical Biophysics, 39: 249-258.

I.C. Baianu: 1983, Natural Transformation Models in Molecular Biology., inProceedings of the SIAM Natl. Meet., Denver, CO.; 1 .

I.C. Baianu: 1984, A Molecular-Set-Variable Model of Structural and Regulatory Activities in Metabolic and Genetic Networks., FASEB Proceedings 43, 917.

I.C. Baianu: 1987a, Computer Models and Automata Theory in Biology and Medicine., in M. Witten (ed.), Mathematical Models in Medicine, vol. 7., Pergamon Press, New York, 1513-1577; 1.

I.C. Baianu: 1987b, Molecular Models of Genetic and Organismic Structures, inProceed. Relational Biology Symp. Argentina; 1.

I.C. Baianu, Glazebrook, J. F. and G. Georgescu: 2004, Categories of Quantum Automata and N-Valued Łukasiewicz Algebras in Relation to Dynamic Bionetworks, (M,R)-Systems and Their Higher Dimensional Algebra, 1 and 1

R. Brown, J. F. Glazebrook and I. C. Baianu: A categorical and higher dimensional algebra framework for complex systems and spacetime structures,Axiomathes 17:409-493. (2007).

L. Löfgren: 1968. On Axiomatic Explanation of Complete SelfReproduction.Bull. Math. Biophysics, 30: 317-348. 
Baianu, I.C.: 2004b Łukasiewicz-Topos Models of Neural Networks, Cell Genome and Interactome Nonlinear Dynamics). CERN Preprint EXT-2004-059. Health Physics and Radiation Effects (June 29, 2004).

Baianu, I. C.: 2006, Robert Rosen's Work and Complex Systems Biology,Axiomathes 16(1-2):25-34.

Baianu I. C., Brown R., Georgescu G. and J. F. Glazebrook: 2006, Complex Nonlinear Biodynamics in Categories, Higher Dimensional Algebra and Lukasiewicz-Moisil Topos: Transformations of Neuronal, Genetic and Neoplastic Networks., Axiomathes, 16 Nos. 1-2: 65-122. 